\title{
Tendências temporais no consumo de tabaco nas capitais brasileiras, segundo dados do VIGITEL, 2006 a 2011
}

\author{
Trends in tobacco consumption from 2006 to \\ 2011 in Brazilian capitals according to the \\ VIGITEL survey \\ Tendencias temporales en el consumo de tabaco \\ en las capitales brasileñas, según datos de \\ VIGITEL, 2006 a 2011
}

Deborah Carvalho Malta 1,2 Betine Pinto Moehlecke Iser 1 Naiza Nayla Bandeira de Sá 1 Renata Tiene de Carvalho Yokota 1 Lenildo de Moura 1

Rafael Moreira Claro 2,3

Micheline Gomes Campos da Luz 1 Regina Ivata Tomie Bernal 3

\begin{abstract}
1 Secretaria de Vigilância em Saúde, Ministério da Saúde, Brasília, Brasil.

2 Escola de Enfermagem Universidade Federal de Minas Gerais, Belo Horizonte, Brasil.

${ }^{3}$ Núcleo de Pesquisas

Epidemiológicas em Nutrição e Saúde, Universidade de São Paulo, São Paulo, Brasil.

Correspondência D. C. Malta

Departamento de Vigilância de Doenças e Agravos Não

Transmissiveis e Promoção da Saúde, Secretaria de

Vigilância em Saúde,

Ministério da Saúde.

The aim of this study was to analyze trends in indicators of smoking in Brazilian State capitals, according to the Surveillance System for Risk and Protective Factors for Chronic Illnesses Using a Telephone Survey (VIGITEL) in adults, from 2006 to 2011. A simple linear regression model was used ( $\alpha=5 \%$ ). There was a decrease in the prevalence of smokers and heavy smokers among men and in individuals 35 to 54 years of age. Smoking also decreased among individuals with 9-11 years of schooling and in the Northeast, North, and Central West regions. For heavy smokers, the largest decline was in the Northeast. Brazil's regulatory policy has been responsible for the decline in tobacco prevalence.
\end{abstract}

SAF Sul - Trecho 02. Lotes 05 e 06. Edifício Premium, bloco F, Torre 1, térreo, sala 16. Brasília, DF 70070-600 Brasil.

damalta@uol.com.br

\section{Abstract}

Smoking; Tobacco Smoke Pollution; Interview; Adult

\section{Resumo}

O objetivo foi analisar a tendência de indicadores do tabagismo nas capitais brasileiras, segundo dados do Sistema de Vigilância de Fatores de Risco e Proteção para Doenças Crônicas por Inquérito Telefônico (VIGITEL) em adultos, 2006 a 2011. Foi utilizado modelo de regressão linear simples ( $\alpha=5 \%$ ). Houve redução da prevalência de fumantes e fumantes pesados entre homens $e$ entre indivíduos com idade de 35 a 54 anos. Para fumantes, também houve redução no estrato de 9 a 11 anos de estudo e regiões Norte e Centro-oeste. Para fumantes pesados, a queda foi na Região Nordeste. A política regulatória adotada pelo Brasil tem sido responsável pelo declínio nas prevalências do tabaco.

Tabagismo; Poluição por Fumaça de Tabaco; Entrevista; Adulto 


\section{Introdução}

As doenças crônicas não transmissíveis (DCNT) respondem por aproximadamente $60 \%$ das mortes ocorridas no mundo e por $72 \%$ daquelas ocorridas no Brasil. Dentre os principais fatores de risco para a ocorrência dessas doenças, destacam-se diversas condições modificáveis, como o tabagismo, o consumo excessivo de álcool, a obesidade, práticas inadequadas de alimentação e o sedentarismo 1,2 .

O consumo de tabaco destaca-se como um dos principais fatores de risco das DCNT, e estimativas da Organização Mundial da Saúde (OMS) apontam cerca de seis milhões de mortes anuais decorrentes do uso direto da erva ou pelo fumo passivo ${ }^{3}$. Estima-se que o tabagismo seja responsável por, aproximadamente, $70 \%$ dos cânceres de pulmão, $42 \%$ das doenças respiratórias crônicas e por cerca de $10 \%$ das doenças do aparelho circulatório ${ }^{4}$. No Brasil, estudo recente sobre carga das doenças relacionadas ao tabaco apontou que cerca de $13 \%$ das mortes, em especial as relacionadas às DCNT, são atribuídas à erva, gerando um custo anual de cerca de 21 bilhões de reais ao Sistema Único de Saúde (SUS) 5.

Os estudos sobre malefícios do fumo remontam à década de 19506 e, até este momento, já acumulam evidências convincentes sobre seus riscos à saúde, inclusive entre as pessoas que convivem com fumantes (fumantes passivos) 7,8 . A OMS destaca algumas das ações protetoras ao uso do tabaco como as intervenções mais custoefetivas na prevenção de DCNT, destacando-se o aumento de impostos e preços sobre os produtos do tabaco 2; a proibição do fumo em lugares públicos 6,8; as advertências sobre os perigos do consumo de tabaco 2; a proibição da propaganda, do patrocínio e da promoção de tabaco 9 .

Existe grande comprometimento internacional com o tema. O tratado internacional da OMS, a Convenção-Quadro para o Controle do Tabaco, já teve adesão de 176 estados-membros 10. Assim, diversos países vêm adotando medidas regulatórias, resultando em redução nas prevalências do tabaco em diversos locais, como Japão, Estados Unidos, Reino Unido e Uruguai, entre outros 11,12 .

No Brasil, a frequência de tabagismo vem sendo medida em diferentes inquéritos domiciliares e, em que pesem diferenças metodológicas no delineamento e tamanho amostral, todos os estudos apontam redução importante nas últimas décadas. No primeiro inquérito, a Pesquisa Nacional em Saúde e Nutrição (1989) mostrou prevalência de tabagismo de $34,8 \%$ (adultos de 18 anos ou mais) 13; estudos subsequentes, como a Pesquisa Mundial de Saúde (2003), mostraram redução para 22,4\% 14; na Pesquisa Especial de Tabagismo (PETab) 15, a prevalência para a população de 15 anos ou mais foi de $17,2 \%$. Essa importante redução no país foi o resultado de medidas educativas, preventivas, legislativas e regulatórias 16,17,18.

O monitoramento dos fatores de risco de DCNT, dentre eles o tabagismo, é importante na definição de politicas públicas, o que motivou a implantação do Sistema de Vigilância de Fatores de Risco e Proteção para Doenças Crônicas por Inquérito Telefônico (VIGITEL) em todas as 26 capitais de estados brasileiros e no Distrito Federal. Entre os indicadores monitorados, encontram-se informações sobre tabagismo, uso de álcool, atividade física e alimentação ${ }^{19}$. O monitoramento de indicadores de tabagismo permite a avaliação da efetividade de medidas de proteção ao tabaco adotadas no país, incluindo as ações regulatórias, que visam a evitar o consumo e incentivar a cessação.

Este estudo tem como objetivo analisar a tendência de indicadores relativos à prevalência do tabagismo nas capitais brasileiras.

\section{Métodos}

O estudo analisa dados do VIGITEL para os anos de 2006 a 2011. O sistema foi implantado em 2006 pelo Ministério da Saúde e provê dados anuais representativos da população adulta ( $\geq 18$ anos) residente nas capitais dos 26 estados brasileiros e do Distrito Federal que possuem telefone fixo.

Foram coletadas, anualmente, cerca de duas mil entrevistas telefônicas em cada uma das 26 capitais do Brasil e no Distrito Federal, totalizando aproximadamente 54 mil indivíduos avaliados em cada ano ${ }^{19}$. O sistema VIGITEL estabelece o tamanho mínimo de amostra de duas mil entrevistas telefônicas em cada cidade para que se possa estimar a frequência de qualquer fator de risco na população adulta, com coeficiente de $95 \%$ de confiança e erro amostral de dois pontos percentuais 19 .

A seleção amostral é realizada por meio de amostragem probabilística em dois estágios: (1) sorteio sistemático de cinco mil linhas telefônicas em cada cidade, seguido por novo sorteio e organização de 25 réplicas (subamostras) de 200 linhas; (2) sorteio de um morador adulto $\geq 18$ anos do domicílio para responder a entrevista 19.

As estimativas dos indicadores foram ponderadas levando-se em conta diferenças na probabilidade de seleção de cada entrevistado, caracterizadas de acordo com o número de linhas telefônicas e de adultos residentes no domicílio sorteado, e diferenças na composição sociode- 
mográfica da amostra do VIGITEL em relação à composição da população adulta total de cada cidade, segundo a distribuição censitária do ano 2000 (Instituto Brasileiro de Geografia e Estatística. Censo demográfico 2000. http://www.ibge. gov.br). São atribuídos pesos finais a cada indivíduo entrevistado, o que é resultante da multiplicação dos seguintes fatores: inverso do número de linhas telefônicas no domicílio do entrevistado; número de adultos no domicílio entrevistado; peso pós-estratificação, que visa a igualar a composição sociodemográfica da população adulta da cidade ao censo populacional, segundo os dados de sexo, faixas etárias e escolaridade. Um quarto fator de ponderação é utilizado para as estimativas relativas ao conjunto das $27 \mathrm{ca}$ pitais: a razão entre a proporção de adultos de uma dada cidade pela proporção de adultos das 27 cidades.

O presente estudo analisou dados referentes à frequência de fumantes (indivíduos que relataram "fumar atualmente") em 2011. Foi considerado fumante o indivíduo que respondeu positivamente à questão " $O(A)$ senhor (a) fuma?" [contendo as seguintes opções de resposta: "Sim, diariamente"; "Sim, ocasionalmente (menos que diariamente)"; "Não"], independentemente do número de cigarros, da frequência e da duração do hábito de fumar. Este indicador foi apresentado por capital e por sexo.

Foram realizadas análises de tendência temporal entre 2006 a 2011 para todos os indicadores relacionados ao tabagismo disponíveis no VIGITEL, quais sejam: (i) frequência de fumantes, (ii) frequência de ex-fumantes (indivíduos não fumantes que responderam positivamente à questão “ $O(a)$ senhor(a) já fumou?", independentemente do número de cigarros e da duração do hábito de fumar); (iii) frequência de fumantes com consumo de 20 ou mais cigarros por dia (calculado pelos indivíduos que indicaram consumo de, no mínimo, 20 cigarros/dia na questão: “Quantos cigarros o(a) senhor(a) fuma por dia?”); (iv) frequência de fumantes passivos no domicílio (indivíduos não fumantes que relataram haver, pelo menos, um dos moradores do seu domicílio que costuma fumar dentro de casa, conforme a questão: "Alguma das pessoas que moram com $o$ (a) senhor(a) costuma fumar dentro de casa?"); (v) frequência de fumantes passivos no local de trabalho (indivíduos não fumantes que relataram haver, pelo menos, uma pessoa que costuma fumar no seu ambiente de trabalho, conforme a questão: "Algum colega do trabalho costuma fumar no mesmo ambiente onde o(a) senhor(a) trabalha?"). A série histórica para os indicadores de fumo passivo contém apenas três anos (20092011), período da avaliação desses indicadores.
Na análise de tendência da série temporal, os indicadores selecionados foram estratificados segundo sexo, sendo expressos pela proporção de adultos que responderam "sim" nas variáveis (i), (ii), (iii), (iv), (v), a cada ano do inquérito. A técnica utilizada para estimar a tendência foi o modelo de regressão linear simples, que tem como variável resposta $\left(\mathrm{Y}_{\mathrm{i}}\right)$ a proporção do indicador e como variável explicativa $\left(\mathrm{X}_{\mathrm{i}}\right)$ o tempo (ano do levantamento) 20. O sinal negativo do coeficiente angular $(\beta)$ da reta ajustada pelo modelo indica que a relação entre o indicador e o tempo é decrescente; em caso contrário, a relação é crescente. $\mathrm{O}$ valor do coeficiente angular positivo representa o aumento médio anual na proporção do indicador para cada unidade de tempo; o contrário representa a queda da média anual na proporção. Foram apresentadas as proporções no período de 2006 a 2011 e a tendência expressa pelo coeficiente angular da reta; apresentou-se também o nível de significância da tendência. Valores de $\alpha$ de $5 \%$ foram considerados tendência significativa. Os indicadores com a tendência significativa foram estratificados segundo faixa etária, escolaridade e região do país. Como medidas de adequação do modelo ajustado, foi utilizada a análise de resíduo, o nível de $5 \%$ de significância e o coeficiente de determinação R2 maior ou igual a $70 \%$. Para o processamento dos dados e análises estatísticas, utilizou-se o programa Stata versão 11.1 (Stata Corp., College Station, Estados Unidos).

Este estudo foi aprovado pela Comissão $\mathrm{Na}$ cional de Ética em Pesquisa em Seres Humanos (CONEP). O consentimento livre e esclarecido foi substituído pelo consentimento verbal obtido por ocasião dos contatos telefônicos com os entrevistados.

\section{Resultados}

No presente estudo, verificou-se diferença nas prevalências de tabagismo segundo sexo e capital; os homens apresentaram maiores prevalências. A frequência de fumantes no conjunto das capitais do Brasil foi de $14,8 \%$, sendo maior entre os homens $(18,1 \%)$ do que entre as mulheres $(12 \%)$, fato verificado em todas as capitais. A frequência de adultos fumantes variou de $7,8 \%$, em Maceió (Alagoas), a 22,6\%, em Porto Alegre (Rio Grande do Sul). Entre homens, as maiores frequências de fumantes foram encontradas em Porto Alegre (24,6\%), Curitiba (Paraná; $24,4 \%$ ) e São Paulo (22,2\%); entre mulheres, em Porto Alegre (20,9\%), São Paulo (16,8\%) e Curitiba (16,5\%). As menores frequências de fumantes no sexo masculino ocorreram em Salvador (Bahia; 
$10,6 \%)$, Maceió (10,9\%) e Recife (Pernambuco; 13,1\%); no sexo feminino, em Aracaju (Sergipe; 4,3\%), Maceió (5,3\%) e João Pessoa (Paraíba; 6\%) (Tabela 1).

$\mathrm{Na}$ análise de tendência, a prevalência de fumantes no Brasil apresentou redução relativa de $0,34 \%$ a cada ano do inquérito, variando de $16,2 \%$ (IC95\%: 15,4-16,9), em 2006, a 14,8\% (IC95\%: 13,9-15,7), em 2011. No sexo masculino, a redução, no período entre 2006 e 2011, ocorreu a uma taxa média de 0,6 pontos percentuais (p.p.) ao ano. Igualmente, a frequência de homens que relataram consumo de 20 cigarros ou mais por dia também diminuiu, em média, em 0,2 p.p. ao ano $(\mathrm{p}<0,05)$. Os indicadores de fumo passivo no trabalho, passivo no domicílio e ex-fumante mantiveram-se estáveis no período (Figura 1a). Não foram verificadas tendências significativas para os indicadores de tabagismo entre as mulheres no período estudado (Figura 1b).

Na análise detalhada do indicador de consumo de tabaco no período, as maiores frequências nas capitais do país ocorrem entre indivíduos do sexo masculino, com idade entre 45 a 54 anos, commenor escolaridade ( 0 a 8 anos de estudo), residentes nas regiões Sul e Sudeste. Em oposição,

Tabela 1

Percentual * de adultos ( $\geq 18$ anos) fumantes, por sexo, segundo as capitais dos estados brasileiros e Distrito Federal. Sistema de Vigilância de Fatores de Risco e Proteção para Doenças Crônicas por Inquérito Telefônico (VIGITEL), 2011.

\begin{tabular}{|c|c|c|c|c|c|c|}
\hline \multirow[t]{3}{*}{ Capitais e Distrito Federal } & \multicolumn{2}{|c|}{ Total } & \multicolumn{4}{|c|}{ Sexo } \\
\hline & \multirow[b]{2}{*}{$\%$} & \multirow[b]{2}{*}{ IC95\% } & \multicolumn{2}{|c|}{ Masculino } & \multicolumn{2}{|c|}{ Feminino } \\
\hline & & & $\%$ & IC95\% & $\%$ & IC95\% \\
\hline Aracaju & 9,4 & $6,9-11,9$ & 15,6 & $10,6-20,5$ & 4,3 & $2,6-6,1$ \\
\hline Belém & 12,5 & $9,2-15,8$ & 19,3 & $13,1-25,5$ & 6,6 & $4,2-9,1$ \\
\hline Belo Horizonte & 15,6 & $12,9-18,2$ & 20,1 & $15,7-24,5$ & 11,7 & $8,6-14,9$ \\
\hline Boa Vista & 13,0 & $9,0-17,0$ & 18,1 & $11,4-24,8$ & 8,0 & $3,6-12,4$ \\
\hline Campo Grande & 13,2 & $10,3-16,1$ & 18,8 & $13,5-24,1$ & 8,1 & $5,8-10,4$ \\
\hline Cuiabá & 16,1 & $12,8-19,4$ & 21,7 & $16,1-27,2$ & 11,1 & $7,4-14,7$ \\
\hline Curitiba & 20,2 & $17,1-23,3$ & 24,4 & $20,0-28,8$ & 16,5 & $12,2-20,8$ \\
\hline Florianópolis & 14,3 & $11,9-16,7$ & 17,5 & $13,4-21,6$ & 11,5 & $8,8-14,2$ \\
\hline Fortaleza & 10,3 & $7,6-13,1$ & 13,5 & $8,6-18,5$ & 7,7 & $4,8-10,6$ \\
\hline Goiânia & 11,0 & $8,7-13,4$ & 16,0 & $11,7-20,4$ & 6,7 & $4,9-8,5$ \\
\hline João Pessoa & 9,4 & $7,1-11,6$ & 13,5 & $9,3-17,7$ & 6,0 & $3,9-8,0$ \\
\hline Macapá & 10,9 & $8,1-13,7$ & 14,9 & $10,1-19,8$ & 7,2 & $4,3-10,1$ \\
\hline Maceió & 7,8 & $5,4-10,2$ & 10,9 & $6,3-15,5$ & 5,3 & $3,3-7,3$ \\
\hline Manaus & 11,9 & $9,1-14,7$ & 16,7 & $12,0-21,3$ & 7,5 & $4,4-10,6$ \\
\hline Natal & 11,4 & $7,6-15,3$ & 17,8 & $10,0-25,5$ & 6,2 & $4,4-8,0$ \\
\hline Palmas & 12,5 & $8,9-16,1$ & 16,4 & $10,7-22,1$ & 8,6 & $4,0-13,2$ \\
\hline Porto Alegre & 22,6 & $19,9-25,3$ & 24,6 & $20,4-28,9$ & 20,9 & $17,4-24,3$ \\
\hline Porto Velho & 16,6 & $13,2-20,0$ & 21,8 & $16,4-27,3$ & 11,4 & $7,5-15,4$ \\
\hline Recife & 12,3 & $9,6-15,1$ & 13,1 & $8,9-17,3$ & 11,7 & $8,1-15,3$ \\
\hline Rio Branco & 14,5 & $11,2-17,8$ & 21,0 & $14,8-27,1$ & 8,6 & $6,1-11,1$ \\
\hline Rio de Janeiro & 14,1 & $11,4-16,7$ & 15,5 & $10,8-20,2$ & 12,9 & $10,0-15,8$ \\
\hline Salvador & 8,6 & $5,9-11,2$ & 10,6 & $6,5-14,8$ & 6,8 & $3,4-10,2$ \\
\hline São Luís & 13,2 & $9,4-16,9$ & 21,6 & $14,7-28,5$ & 6,2 & $3,2-9,1$ \\
\hline São Paulo & 19,3 & $16,5-22,1$ & 22,2 & $18,1-26,4$ & 16,8 & $13,0-20,6$ \\
\hline Teresina & 15,3 & $11,2-19,4$ & 19,2 & $12,8-25,7$ & 12,1 & $6,9-17,3$ \\
\hline Vitória & 11,2 & $8,7-13,7$ & 15,2 & $10,9-19,6$ & 7,8 & $5,0-10,7$ \\
\hline Distrito Federal & 13,5 & $10,1-16,9$ & 14,2 & $9,1-19,3$ & 12,8 & $8,3-17,4$ \\
\hline Conjuntos das capitais & 14,8 & $13,9-15,7$ & 18,1 & $16,6-19,5$ & 12,0 & $10,8-13,1$ \\
\hline
\end{tabular}

IC95\%: Intervalo de 95\% de confiança.

* Percentual ponderado para ajustar a distribuição sociodemográfica da amostra do VIGITEL à distribuição da população adulta de cada cidade no Censo Demográfico de 2000 (Instituto Brasileiro de Geografia e Estatística. http://www.ibge.gov.br). 
Variação temporal de indicadores de tabagismo e da população adulta masculina (1a) e feminina (1b) das capitais dos estados brasileiros e Distrito Federal. Sistema de Vigilância de Fatores de Risco e Proteção para Doenças Crônicas por Inquérito Telefônico (VIGITEL), 2006-2011.
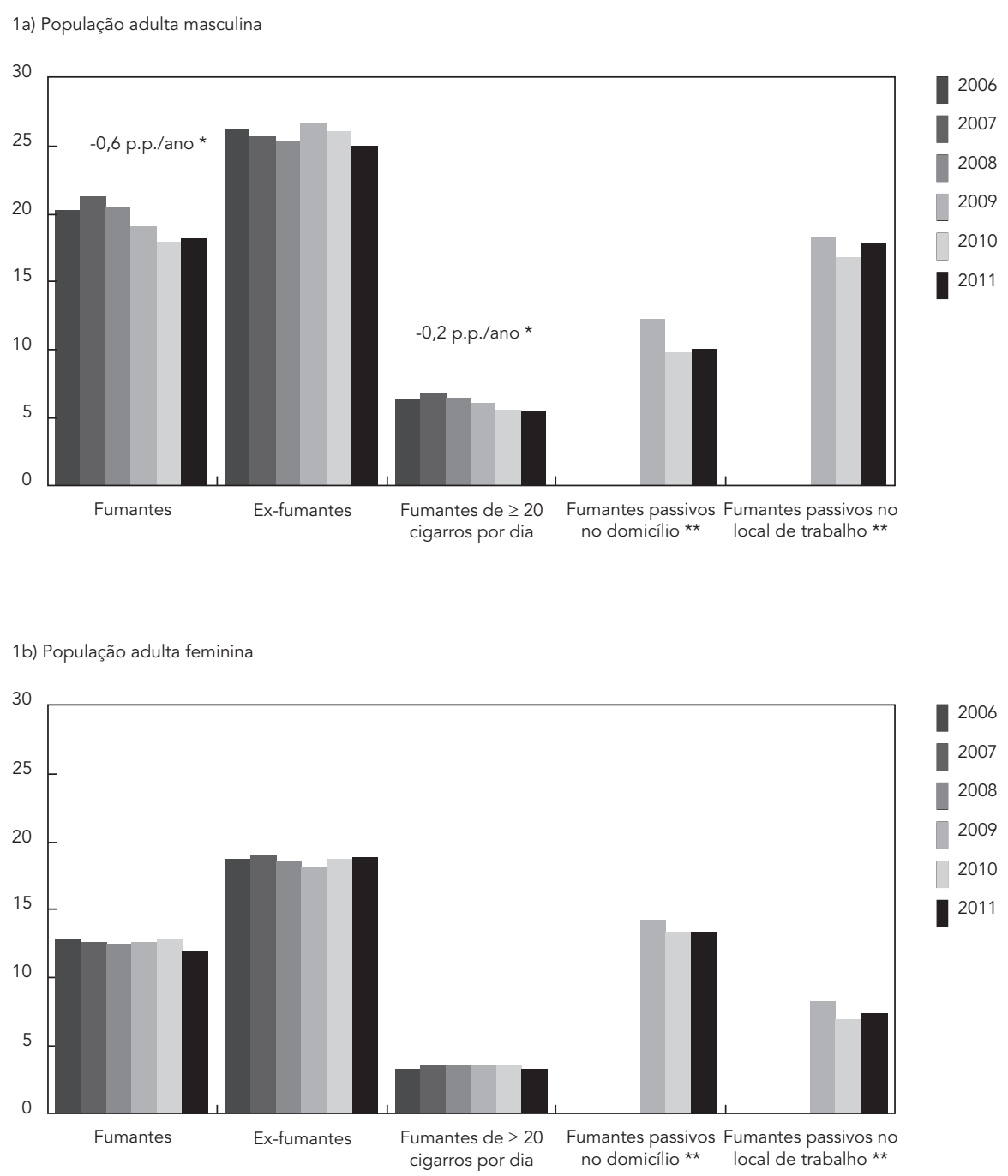

\footnotetext{
* Correspondente ao coeficiente da regressão linear do valor do indicador sobre o ano do levantamento, valor de $p<0,05$

** Indicadores de fumo passivo no domicílio e no local de trabalho foram incluídos no VIGITEL em 2009
}

menores prevalências ocorrem em mulheres pessoas de 65 anos ou mais, com mais de nove anos de estudo, residentes nas regiões Norte e Nordeste (Tabela 2). As análises de tendência estratificadas segundo faixa etária, escolaridade $e$ região do país indicaram redução na frequência de fumantes nas faixas de 35 a 44 anos e de 45 a 54 anos, entre os indivíduos de escolaridade intermediária (9 a 11 anos) e entre os residen- tes nas capitais das regiões Norte e Centro-oeste (Tabela 2).

A análise detalhada do indicador consumo de 20 cigarros apresentou padrão semelhante ao verificado para o consumo de tabaco, e a tendência no período 2006-2011 indicou redução na população com idade entre 35 a 54 anos e na Região Nordeste, sem redução nas prevalências das demais regiões (Tabela 3 ). 
Tabela 2

Análise de tendência de fumantes nas capitais dos estados brasileiros e Distrito Federal (2006-2011), segundo faixa etária, escolaridade e região. Sistema de Vigilância de Fatores de Risco e Proteção para Doenças Crônicas por Inquérito Telefônico (VIGITEL), 2006-2011.

\begin{tabular}{|c|c|c|c|c|c|c|c|c|c|c|c|c|c|c|}
\hline \multirow[t]{2}{*}{ Variável } & \multicolumn{2}{|r|}{2006} & \multicolumn{2}{|r|}{2007} & \multicolumn{2}{|r|}{2008} & \multicolumn{2}{|c|}{2009} & \multicolumn{2}{|r|}{2010} & \multicolumn{2}{|r|}{2011} & \multirow{2}{*}{$\begin{array}{l}\text { Coeficiente } \\
\text { de regressão }\end{array}$} & \multirow{2}{*}{$\begin{array}{l}\text { Valor } \\
\text { de } p \text { * }\end{array}$} \\
\hline & $\%$ & IC95\% & $\%$ & IC95\% & $\%$ & IC95\% & $\%$ & IC95\% & $\%$ & IC95\% & $\%$ & IC95\% & & \\
\hline Total & 16,2 & $15,4-16,9$ & 16,6 & $15,7-17,4$ & 16,1 & $15,0-17,3$ & 15,5 & $14,5-16,5$ & 15,1 & $14,2-16,0$ & 14,8 & $13,9-15,7$ & $-0,3457$ & 0,008 \\
\hline \multicolumn{15}{|l|}{ Faixa etária (anos) } \\
\hline $18-24$ & 14,3 & $11,9-16,7$ & 16,9 & $14,2-19,5$ & 16,5 & $12,0-20,9$ & 15,0 & $11,5-18,5$ & 12,5 & $10,5-14,6$ & 12,5 & $9,9-15,2$ & $-0,6771$ & 0,147 \\
\hline $25-34$ & 14,8 & $13,3-16,2$ & 15,7 & $14,0-17,3$ & 15,6 & $13,7-17,5$ & 16,4 & $14,4-18,4$ & 17,3 & $15,0-19,6$ & 16,1 & $14,0-18,2$ & 0,3457 & 0,075 \\
\hline $35-44$ & 18,8 & $17,3-20,4$ & 17,4 & $16,0-18,8$ & 16,4 & $15,0-17,8$ & 15,1 & $13,6-16,6$ & 15,2 & $13,7-16,8$ & 14,5 & $12,9-16,1$ & $-0,8400$ & 0,002 \\
\hline $45-54$ & 21,9 & $20,1-23,6$ & 21,7 & $19,8-23,5$ & 19,7 & $18,0-21,4$ & 19,0 & $17,3-20,8$ & 18,3 & $16,4-20,2$ & 19,0 & $17,3-20,8$ & $-0,7257$ & 0,015 \\
\hline $55-64$ & 15,4 & $13,5-17,4$ & 16,2 & $14,2-18,2$ & 17,1 & $15,1-19,2$ & 16,9 & $14,9-18,9$ & 16,5 & $14,3-18,7$ & 16,2 & $14,3-18,1$ & 0,1343 & 0,413 \\
\hline$\geq 65$ & 9,3 & $7,8-10,7$ & 8,1 & $6,6-9,6$ & 9,1 & $7,6-10,6$ & 8,1 & $6,8-9,4$ & 8,1 & $6,7-9,5$ & 8,7 & $7,1-10,3$ & $-0,1143$ & 0,552 \\
\hline \multicolumn{15}{|c|}{ Escolaridade (anos } \\
\hline \multicolumn{15}{|l|}{ de estudo) } \\
\hline $0-8$ & 19,1 & $17,8-20,5$ & 19,7 & $18,2-21,1$ & 20,1 & $18,1-22,2$ & 19,3 & $17,5-21,1$ & 18,6 & $17,1-20,2$ & 18,8 & $17,2-20,4$ & $-0,1600$ & 0,276 \\
\hline $9-11$ & 13,4 & $12,5-14,3$ & 13,0 & $12,1-13,9$ & 11,6 & $10,7-12,4$ & 11,3 & $10,5-12,2$ & 11,6 & $10,6-12,5$ & 10,1 & $9,2-10,9$ & $-0,6000$ & 0,006 \\
\hline$\geq 12$ & 11,5 & $10,4-12,7$ & 12,9 & $11,7-14,1$ & 11,5 & $10,4-12,6$ & 11,1 & $10,0-12,2$ & 10,2 & $9,2-11,3$ & 10,3 & $9,2-11,5$ & $-0,4143$ & 0,064 \\
\hline \multicolumn{15}{|l|}{ Região } \\
\hline Nordeste & 13,9 & $12,9-14,8$ & 13,6 & $12,5-14,6$ & 11,0 & $10,1-11,9$ & 13,0 & $11,8-14,2$ & 11,1 & $10,1-12,2$ & 10,6 & $9,5-11,7$ & $-0,6286$ & 0,054 \\
\hline Norte & 15,7 & $14,4-17,0$ & 15,8 & $14,4-17,2$ & 14,4 & $12,9-15,9$ & 13,4 & $11,9-14,8$ & 14,1 & $12,4-15,9$ & 12,6 & $11,0-14,2$ & $-0,6171$ & 0,010 \\
\hline Sudeste & 17,0 & $15,5-18,6$ & 18,2 & $16,6-19,9$ & 19,3 & $16,9-21,6$ & 16,6 & $14,7-18,6$ & 17,2 & $15,6-18,9$ & 17,1 & $15,3-18,9$ & $-0,1486$ & 0,594 \\
\hline Sul & 19,8 & $18,0-21,5$ & 19,7 & $18,0-21,3$ & 18,6 & $17,0-20,2$ & 20,7 & $19,0-22,4$ & 18,1 & $16,4-19,8$ & 20,5 & $18,6-22,4$ & 0,0229 & 0,938 \\
\hline Centro-oeste & 16,0 & $14,3-17,8$ & 15,4 & $13,8-17,0$ & 15,5 & $13,8-17,2$ & 15,2 & $12,8-17,7$ & 14,5 & $12,0-17,0$ & 13,1 & $11,3-14,9$ & $-0,5000$ & 0,012 \\
\hline
\end{tabular}

* Correspondente ao coeficiente da regressão linear do valor do indicador sobre o ano do levantamento, em cada categoria de análise.

Vale destacar que as recusas à participação nas entrevistas do VIGITEL são monitoradas anualmente e têm sido declinantes: 9,1\% (2006); $7,7 \%$ (2007); 5,8\% (2008); 3\% (2009); 2,3\% (2010) e $2,2 \%$ (2011).

\section{Discussão}

O estudo possibilitou analisar dados do VIGITEL entre adultos residentes nas capitais brasileiras e detentores de telefone fixo, nos últimos seis anos, apontando as tendências mais recentes de consumo de tabaco no país. Houve redução da prevalência de fumantes e fumantes pesados entre homens e entre indivíduos com idade de 35 a 54 anos. No caso de fumantes, ocorreu ainda redução entre aqueles com escolaridade de 9 a 11 anos de estudo, residentes nas capitais do Norte e Centro-oeste. Para fumantes pesados, a redução ocorreu apenas na Região Nordeste.
O limite do estudo refere-se ao uso do cadastro de telefones fixos nas capitais para fins de sorteio da amostra. Nas regiões metropolitanas do Sul, Sudeste e Centro-oeste com cobertura acima de $70 \%$, os vícios decorrentes da exclusão de domicílios sem telefone fixo podem ser considerados desprezíveis 21. Entretanto, as regiões Norte e Nordeste apresentam taxas de cobertura inferiores a $70 \%$. Para corrigir os vícios potencias decorrentes da baixa cobertura de domicílios com telefone fixo, o VIGITEL utiliza o método de ponderação por célula segundo sexo, idade e escolaridade, ajustando a distribuição da amostra às características da população residente em cada capital, de acordo com os dados de censo do Instituto Brasileiro de Geografia e Estatística (IBGE), reduzindo-se, assim, as diferenças entre a população com e sem telefone 19. Esse método permite corrigir o vício potencial na prevalência de fumantes em região com baixa cobertura 22 . Outros estudos comparativos entre os dados do 
Análise de tendência do consumo de 20 ou mais cigarros/dia nas capitais dos estados brasileiros e Distrito Federal (2006-2011), segundo faixa etária, escolaridade e região. Sistema de Vigilância de Fatores de Risco e Proteção para Doenças Crônicas por Inquérito Telefônico (VIGITEL), $2006-2011$.

\begin{tabular}{|c|c|c|c|c|c|c|c|c|c|c|c|c|c|c|}
\hline \multirow[t]{2}{*}{ Variável } & \multicolumn{2}{|c|}{2006} & \multicolumn{2}{|c|}{2007} & \multicolumn{2}{|c|}{2008} & \multicolumn{2}{|c|}{2009} & \multicolumn{2}{|c|}{2010} & \multicolumn{2}{|c|}{2011} & \multirow{2}{*}{$\begin{array}{l}\text { Coeficiente } \\
\text { de regressão }\end{array}$} & \multirow{2}{*}{$\begin{array}{l}\text { Valor } \\
\text { de } p \text { * }\end{array}$} \\
\hline & $\%$ & IC95\% & $\%$ & IC95\% & $\%$ & IC95\% & $\%$ & IC95\% & $\%$ & IC95\% & $\%$ & IC95\% & & \\
\hline Total & 4,6 & $4,3-5,0$ & 5,0 & $4,5-5,5$ & 4,9 & $4,4-5,4$ & 4,7 & $3,9-5,5$ & 4,5 & $4,0-5,0$ & 4,3 & $3,8-4,8$ & $-0,0914$ & 0,152 \\
\hline \multicolumn{15}{|l|}{ Faixa etária (anos) } \\
\hline $18-24$ & 2,7 & $1,9-3,4$ & 3,9 & $2,3-5,5$ & 3,4 & $2,0-4,8$ & 4,4 & $1,2-7,6$ & 2,8 & $1,7-4,0$ & 2,5 & $1,5-3,4$ & $-0,0943$ & 0,655 \\
\hline $25-34$ & 3,2 & $2,5-3,9$ & 4,2 & $3,2-5,2$ & 4,1 & $3,1-5,1$ & 3,6 & $2,5-4,7$ & 4,3 & $3,3-5,4$ & 4,1 & $2,7-5,4$ & 0,1229 & 0,270 \\
\hline $35-44$ & 5,7 & $4,9-6,5$ & 5,3 & $4,4-6,1$ & 5,2 & $4,3-6,0$ & 5,3 & $4,3-6,3$ & 4,5 & $3,6-5,4$ & 4,0 & $3,1-4,9$ & $-0,3086$ & 0,009 \\
\hline $45-54$ & 9,0 & $7,7-10,2$ & 8,1 & $6,9-9,4$ & 7,4 & $6,2-8,5$ & 6,6 & $5,5-7,8$ & 7,0 & $5,7-8,3$ & 7,0 & $5,9-8,2$ & $-0,4029$ & 0,032 \\
\hline $55-64$ & 5,8 & $4,4-7,2$ & 6,9 & $5,3-8,4$ & 7,4 & $5,9-8,9$ & 6,6 & $5,1-8,1$ & 6,9 & $5,1-8,7$ & 6,0 & $4,8-7,2$ & 0,0057 & 0,973 \\
\hline$\geq 65$ & 2,5 & $1,8-3,2$ & 2,4 & $1,6-3,2$ & 3,9 & $2,8-5,1$ & 1,9 & $1,4-2,4$ & 2,4 & $1,6-3,1$ & 3,7 & $2,4-5,0$ & 0,1143 & 0,611 \\
\hline \multicolumn{15}{|c|}{ Escolaridade (anos } \\
\hline \multicolumn{15}{|l|}{ de estudo) } \\
\hline $0-8$ & 5,5 & $4,9-6,1$ & 6,2 & $5,3-7,1$ & 6,6 & $5,7-7,4$ & 6,4 & $4,9-7,8$ & 5,6 & $4,8-6,4$ & 5,8 & $4,9-6,6$ & $-0,0143$ & 0,911 \\
\hline $9-11$ & 3,7 & $3,3-4,2$ & 3,6 & $3,2-4,1$ & 2,7 & $2,3-3,1$ & 2,7 & $2,3-3,2$ & 3,3 & $2,8-3,8$ & 2,4 & $2,0-2,7$ & $-0,2114$ & 0,097 \\
\hline$\geq 12$ & 3,4 & $2,8-4,1$ & 3,6 & $3,0-4,2$ & 3,5 & $2,9-4,1$ & 2,9 & $2,3-3,4$ & 3,1 & $2,5-3,7$ & 3,0 & $2,4-3,7$ & $-0,1171$ & 0,079 \\
\hline \multicolumn{15}{|l|}{ Região } \\
\hline Nordeste & 4,0 & $3,5-4,5$ & 3,3 & $2,7-3,9$ & 3,3 & $2,8-3,9$ & 3,0 & $2,6-3,5$ & 2,9 & $2,3-3,4$ & 2,8 & $2,2-3,3$ & $-0,2143$ & 0,009 \\
\hline Norte & 3,3 & $2,7-3,9$ & 3,2 & $2,6-3,8$ & 3,5 & $2,6-4,4$ & 2,9 & $2,2-3,7$ & 2,9 & $2,1-3,7$ & 2,9 & $2,2-3,6$ & $-0,1000$ & 0,099 \\
\hline Sudeste & 5,0 & $4,3-5,7$ & 6,2 & $5,2-7,2$ & 5,9 & $4,9-6,8$ & 5,7 & $4,0-7,3$ & 5,4 & $4,5-6,3$ & 5,2 & $4,2-6,1$ & $-0,0457$ & 0,718 \\
\hline Sul & 7,2 & $6,1-8,3$ & 7,0 & $5,8-8,1$ & 6,9 & $5,9-7,9$ & 7,3 & $6,3-8,4$ & 6,8 & $5,7-7,9$ & 8,0 & $6,7-9,4$ & 0,1086 & 0,349 \\
\hline Centro-oeste & 4,0 & $3,3-4,6$ & 4,6 & $3,6-5,5$ & 4,7 & $3,8-5,7$ & 4,3 & $2,7-5,9$ & 4,6 & $3,5-5,7$ & 3,0 & $2,4-3,7$ & $-0,1543$ & 0,371 \\
\hline
\end{tabular}

* Correspondente ao coeficiente da regressão linear do valor do indicador sobre o ano do levantamento, em cada categoria de análise.

VIGITEL e inquéritos domiciliares apontaram a importância dessas ponderações, que tendem a aproximar-se das estimativas populacionais, embora ainda possam permanecer vícios em algumas variáveis, em especial naquelas associadas à baixa renda e à baixa escolaridade 23,24,25

OVIGITEL também utiliza procedimentos de supervisão e monitoramento da qualidade dos dados, sendo realizada capacitação constante dos entrevistadores, além de checagem e auditorias em cerca de $10 \%$ da produção de cada dia, em amostras aleatórias. Todas as entrevistas são gravadas e, quando necessário, novo contato telefônico é realizado para conferência dos dados. O monitoramento dos dados torna possível a análise de tendências, embora o tempo de análise, seis anos, ainda seja curto, podendo ocorrer mudanças na série histórica.

São ainda acompanhados indicadores como taxas de recusa, taxa de sucesso, percentual de linhas não disponíveis, percentual de entrevistas completas com mulheres e em domicílios com apenas um morador adulto. As taxas atuais de recusa são consideradas desprezíveis, principalmente considerando que estas podem chegar a $30 \%$ em inquéritos semelhantes, como o Behavioral Risk Factor Surveillance System, sistema de vigilância de fatores de risco comportamentais dos Estados Unidos 26 .

O VIGITEL utiliza a escolaridade como proxy de situação socioeconômica, o que se justifica pela simplicidade na coleta dessa informação por telefone. A literatura aponta que renda e escolaridade estão associadas a maiores prevalências do tabaco, podendo subestimar as prevalências aqui descritas 15,27 .

A maior prevalência de tabagismo em pessoas com menor renda e escolaridade também já foi verificada no Brasil 15,27 e em outros países, como na Itália 28 , onde um inquérito apontou maior probabilidade de consumo de cigarros e aderência ao hábito de fumar entre homens com baixa escolaridade. Em Alberta, Canadá, três inquéritos nacionais realizados entre os anos 2000 e 2005 identificaram uma relação inversa entre a prevalência de fumantes e o nível de escolaridade dos entrevistados 29 . Resultados semelhantes foram também encontrados na África do Sul, onde os inquéritos Demographic and Health Surveys apontaram associação direta entre, de um lado, alta renda e educação e, de outro, baixa prevalência de tabagismo 30.

A diferença na frequência de consumo de tabaco entre sexos apontada no presente estudo 
também foi identificada em outros inquéritos no Brasil 11,15 e no mundo 31. Essas diferenças tendem a ser minimizadas pela redução contínua do tabagismo entre homens e em face da estabilidade na frequência observada entre as mulheres. Estudo realizado na França mostrou tendência semelhante: redução, em aproximadamente $40 \%$, do hábito de fumar entre homens, no período de 1980 a 2005-2007, e aumento de cerca de $1 \%$ entre mulheres, no mesmo período, aproximando as prevalências entre os sexos 32 .

O início do uso do tabaco na Europa e nas Américas, no princípio do século 20, deu-se com o sexo masculino, associando-se sempre à ideia de masculinidade, força e poder, fortemente ancorada em propagandas que vendiam a imagem de sucesso ${ }^{33}$. Esse perfil se mantém, e estudo comparativo do Global Adults Tobacco Survey, entre 16 países de média e baixa renda, mostrou elevadas prevalências entre homens; entre mulheres, as frequências tendem a ser mais baixas, dependendo da localidade. Países como Egito e Bangladesh, com forte influência religiosa e culturas repressivas em relação às mulheres, tendem a ter prevalências menores que $2 \%$ entre elas, contrastando com cerca de $30 \%$ entre homens 31 .

Situação semelhante foi encontrada no Brasil. Capitais do Sul e Sudeste, como Porto Alegre, Curitiba e São Paulo, apresentam prevalências de fumo elevadas em mulheres e próximas às dos homens; por sua vez, em diversas cidades do Nordeste e Norte, a prevalência entre homens chega a ser quase três vezes maior. A diferença entre sexos nas capitais do Norte e Nordeste, que ainda deve ser mais bem compreendida, é explicada, em parte, por questões culturais e pela menor participação da mulher na sociedade no passado, em especial no início da introdução do uso do tabaco nestas regiões, semelhante ao que já foi observado em outros países do mundo 34,35,36. Atualmente, o tabaco está fortemente associado às populações de baixa escolaridade, diferente do que se verificava antigamente, quando a erva esteve associada ao movimento de autoafirmação, independência e participação das mulheres na sociedade e no mercado de trabalho, sendo seu uso influenciado pelos meios de comunicação e cinema 37,38 . No Brasil a prevalência do fumo entre mulheres foi crescente a partir das décadas de 60 e 70, em especial nas cidades do Sudeste e Sul, mais ricas e com escolaridade mais elevada entre o sexo feminino. Em anos mais recentes, em virtude de campanhas educativas, medidas regulatórias e restritivas, o tabaco perdeu prestígio e adeptos, levando a uma estabilização das prevalências anteriores elevadas nas capitais do Sul e Sudeste e menores frequências entre mulheres no Norte e Nordeste.

O fato de, nos últimos seis anos, o declínio ter ocorrido apenas entre homens, que representam a grande proporção de fumantes no Norte e Nordeste, também ajuda a explicar o melhor desempenho dessas regiões nas análises de tendência 39. Outras hipóteses, como medidas regulatórias nestas áreas, ainda precisam ser mais bem avaliadas, já que são muito recentes e não foram suficientemente verificadas. Além disso, o Sul apresenta forte influência de populações migrantes europeias, onde o tabaco está fortemente arraigado à cultura local, e de países fronteiriços, como Argentina e Uruguai, nos quais a prevalência chega a mais de $30 \%$. Pesa também o fato de a Região Sul ser grande produtora de fumo, elevando o Brasil ao segundo lugar na produção global e ao primeiro lugar na exportação do tabaco 41 .

Em relação ao consumo passivo de tabaco, a PETab, realizada em 2008, apontou que $24,4 \%$ dos entrevistados relataram respirar fumaça ambiental de tabaco em seus locais de trabalho 15 . A fumaça de cigarros, charutos e similares contamina recintos coletivos, e as evidências demonstram que o fumo passivo também resulta em doenças como câncer e infarto. Não existe grau seguro para essa exposição, nem sistema de ventilação capaz de reduzi-la a níveis aceitáveis 33,42 . A literatura corrobora, ainda, o efeito dose-resposta para o câncer de pulmão e fumo 43 , tornando importante o declínio da frequência de pessoas que fumam mais que 20 cigarros por dia, na redução de DCNT. Torna-se importante avançar também na redução da prevalência do tabaco no sexo feminino, a fim de se obter melhora de indicadores de doenças relacionadas ao tabaco em ambos os sexos 44 .

A redução da prevalência do tabaco no país está relacionada a inúmeros fatores, com destaque para as ações regulatórias, como a proibição de propaganda, da promoção e do patrocínio de cigarros (com exceção dos pontos de venda), da vinculação de imagens de advertências nos maços e pacotes de produtos do tabaco, além da proibição de fumo em ambientes fechados. A adesão do Brasil à Convenção-Quadro para o Controle do Tabaco em 2005, vinculando o país legalmente ao tratado, representa um marco no processo de enfrentamento do tabaco 18. Em 2011, foi aprovada nova lei que regulamenta os ambientes livres de fumo, amplia as advertências nos maços, define preço mínimo de venda e aumenta a taxação dos cigarros para $85 \% 17$. Em 2012, a Agência Nacional de Vigilância Sanitária (ANVISA) aprovou a proibição de aditivos de sabor nos cigarros. A estratégia multissetorial 
tem sido fundamental, como a participação do desenvolvimento agrário, agricultura, educação, planejamento, receita, entre outros. Dessa forma, o Brasil se destaca globalmente pelos avanços na política antitabaco e no declínio das prevalências deste $1,16,45$.

O Plano de Ações Estratégicas para o Enfrentamento de DCNT visa à continuidade da redução do tabagismo no país, tendo como meta che- gar ao patamar de $10 \%$ da população em 2022 Torna-se um desafio reduzir a exposição ativa e passiva ao tabaco entre indivíduos de diferentes estratos populacionais, reduzir a prevalência em populações de baixa escolaridade e renda, mulheres e adultos jovens. Destaca-se a importância do monitoramento anual dos indicadores do tabaco no enfretamento das DCNT 17,19.

\section{Resumen}

El objetivo fue analizar la tendencia de indicadores del tabaquismo en las capitales brasileñas, según datos del Sistema de Vigilancia de Factores de Riesgo y Protección para Enfermedades Crónicas por Encuesta Telefónica (VIGITEL) en adultos, de 2006 a 2011. Fue utilizado el modelo de regresión lineal simple $(\alpha=5 \%)$. Hubo una reducción de la prevalencia de fumadores $y$ fumadores crónicos entre hombres y entre individuos con edad de 35 a 54 años. En los fumadores, también hubo reducción en el estrato de 9 a 11 años de estudio y regiones Norte y Centro-oeste. En los fumadores crónicos, la caída fue en la región Nordeste. La política regulatoria adoptada por Brasil ha sido responsable del declive en las prevalencias del tabaco.

Tabaquismo; Contaminación por Humo de Tabaco; Entrevista; Adulto

\section{Colaboradores}

D. C. Malta foi responsável pela concepção do projeto, delineamento do estudo, análise e interpretação dos dados, revisão da literatura, elaboração da versão inicial e aprovação da versão final do texto. B. P. M. Iser, N. N. B. Sá, R. M. Claro e R. I. T. Bernal foram corresponsáveis pelas análises estatísticas, análise e interpretação dos dados, contribuíram na revisão crítica do artigo e aprovaram a versão final do artigo. R. T. C. Yokota, L. Moura e M. G. C. Luz participaram da análise e interpretação dos dados e contribuíram na revisão crítica do artigo. 


\section{Referências}

1. Schmidt MI, Duncan BB, Azevedo e Silva G, Menezes AM, Monteiro CA, Barreto SM, et al. Chronic non-communicable diseases in Brazil: burden and current challenges. Lancet 2011; 377:1949-61.

2. World Health Organization. Global status report on non-communicable diseases, 2010. Geneva: World Health Organization; 2011.

3. World Health Organization. Global estimate of the burden of disease from second-hand smoke. Geneva: World Health Organization; 2010.

4. World Health Organization. Global health risks: mortality and burden of disease attributable to selected major risks. Geneva: World Health Organization; 2009.

5. Aliança de Controle do Tabagismo. Carga das doenças tabaco-relacionadas para o Brasil. http:// actbr.org.br/uploads/conteudo/721_Relatorio_ Carga_do_tabagismo_Brasil.pdf (acessado em 10/ Out/2012).

6. World Health Organization. Protection from exposure to second-hand tobacco smoke: policy recommendations. Geneva: World Health Organization; 2007.

7. Doll R, Hill AB. Mortality in relation to smoking: ten years' observations of British doctors. BMJ $1964 ; 1: 1460-7$

8. International Agency for Research on Cancer. Evaluating the effectiveness of smoke-free policies. Lyon: IARC Press; 2009.

9. Jamison DT, Breman JG, Measham AR, Alleyne G, Claeson M, Evans DB, et al. Disease control priorities in developing countries. 2nd Ed. Washington DC: World Bank; 2006.

10. Araujo AJ, Silva LCC. Diversificação de cultivos: solução para a fumicultura. http://www.sbpt. org.br/?op=itLeitura\&id srv=2\&id tpc $=0$ \&nid tp c $=\& i d_{-}$gr p $=1 \&$ add $=\& 1 \mathrm{k}=1 \& \mathrm{nti}=765 \& \mathrm{l}_{-}$ $n t i=S \& i t g=S \& s t=\& d s t=3 \quad$ (acessado em 20/ Out/2012).

11. Monteiro CA, Cavalcante TM, Moura EC, Claro RM, Szwarcwald CL. Population-based evidence of a strong decline in the prevalence of smokers in Brazil (1989-2003). Bull World Health Organ 2007; 85:527-34

12. Sebrié EM, Sandoya E, Hyland A, Bianco E, Glantz SA, Cummings KM. Hospital admissions for acute myocardial infarction before and after implementation of a comprehensive smoke-free policy in Uruguay. Tob Control 2012; [Epub ahead of print].

13. Instituto Nacional de Alimentação e Nutrição. Pesquisa Nacional sobre Saúde e Nutrição (PNSN) 1989. Brasília: Ministério da Saúde; 1990.

14. Szwarcwald CL, Viacava F, Vasconcellos MTL, Leal MC, Azevedo LO, Queiroz RSB, et al. Pesquisa Mundial de Saúde 2003: o Brasil em números. RADIS Comunicação em Saúde 2004; 23:14-33.

15. Instituto Brasileiro de Geografia e Estatística. Pesquisa Especial de Tabagismo (PETab). Rio de Janeiro: Instituto Brasileiro de Geografia e Estatística; 2009.
16. Ministério da Saúde. Plano de ações estratégicas para o enfrentamento das doenças crônicas não transmissíveis (DCNT) no Brasil 2011-2022. Brasília: Ministério da Saúde; 2011.

17. Malta DC, Morais Neto OL, Silva Junior JB; Grupo Técnico de Redação. Apresentação do plano de ações estratégicas para o enfrentamento das doenças crônicas não transmissíveis no Brasil, 2011 a 2022. Epidemiol Serv Saúde 2011; 20:425-38.

18. Presidência da República. Decreto no 5.658 , de 2 de janeiro de 2006. Diário Oficial da União 2006; 3 jan.

19. Departamento de Análise de Situação de Saúde, Secretaria de Vigilância em Saúde, Ministério da Saúde. Vigilância de fatores de risco e proteção para doenças crônicas por inquérito telefônico: VIGITEL 2011. Brasília: Ministério da Saúde; 2012.

20. Morettin PA, Toloi CMC. Análise de séries temporais. 2a Ed. São Paulo: Edgard Blücher; 2006.

21. Bernal R, Silva NN. Cobertura de linhas telefônicas residenciais e vícios potenciais em estudos epidemiológicos. Rev Saúde Pública 2009; 43:421-6.

22. Bernal RTI. Inquéritos por telefone: inferências válidas em regiões com baixa taxa de cobertura de linhas residenciais [Tese de Doutorado]. São Paulo: Universidade de São Paulo; 2011.

23. Francisco PMSB, Barros MBA, Segri NJ, Alves MCGP, Cesar CLG, Malta DC. Comparação de estimativas para o auto-relato de condições crônicas entre inquérito domiciliar e telefônico - Campinas (SP), Brasil. Rev Bras Epidemiol 2011; 14 Suppl 1:515.

24. Ferreira AD, Cesar CC, Malta DC, Andrade AC, Ramos CG, Proietti FA, et al. Validade de estimativas obtidas por inquérito telefônico: comparação entre VIGITEL 2008 e inquérito Saúde em Beagá. Rev Bras Epidemiol 2011; 14 Suppl 1:16-30.

25. Segri NJ, Francisco PM, Alves MC, Barros MB, Cesar CL, Goldbaum M, et al. Práticas preventivas de detecção de câncer em mulheres: comparação das estimativas dos inquéritos de saúde (ISA-Capital) e vigilância de fatores de risco e proteção para doenças crônicas por inquérito telefônico (VIGITEL - São Paulo). Rev Bras Epidemiol 2011; 14 Suppl 1:31-43.

26. Centers for Disease Control and Prevention. Behavioral risk factor surveillance system: 2010 summary data quality report. ftp://ftp.cdc.gov/pub/ Data/Brfss/2010_Summary_Data_Quality_Report. pdf (acessado em 10/Out/2012).

27. Opaleye ES, Sanchez ZM, Moura YG, Galduróz JCF, Locatelli DP, Noto AR. The Brazilian smoker: a survey in the largest cities of Brazil. Rev Bras Psiquiatr 2012; 34:43-51.

28. Sardu C, Mereu A, Minerba L, Contu P. The Italian national trends in smoking initiation and cessation according to gender and education. J Prev Med Hyg 2009; 50:191-5. 
29. Li FX, Robson PJ, Ashbury FD, Hatcher J, Bryan HE. Smoking frequency, prevalence and trends, and their socio-demographic associations in Alberta, Canada. Can J Public Health 2009; 100:453-8.

30. Peer N, Bradshaw D, Laubscher R, Steyn K. Trends in adult tobacco use from two South African Demographic and Health Surveys conducted in 1998 and 2003. S Afr Med J 2009; 99:744-9.

31. Giovino GA, Mirza SA, Samet JM, Gupta PC, Jarvis MJ, Bhala N, et al. Tobacco use in 3 billion individuals from 16 countries: an analysis of nationally representative cross-sectional household surveys. Lancet 2012; 380:668-79.

32. Tilloy E, Cottel D, Ruidavets J-B, Arveiler D, Ducimetière $\mathrm{P}$, Bongard $\mathrm{V}$, et al. Characteristics of current smokers, former smokers, and second-hand exposure and evolution between 1985 and 2007. Eur J Cardiovasc Prev Rehabil 2010; 17:730-6.

33. Musk AW, Klerk NH. History of tobacco and health. Respirology 2003; 8:286-90.

34. Amos A, Haglund M. From social taboo to "torch of freedom": the marketing of cigarettes to women. Tob Control 2000; 9:3-8.

35. Apelberg B, Aghi M, Asma S, Donaldson E, Yeong CC, Vaithinathan R. Prevalence of tobacco use and factors influencing initiation and maintenance among women. In: Samet JM, Soon-Young Y, editors. Gender, women, and the tobacco epidemic. Geneva: World Health Organization; 2010. p. 29-50.

36. Centers for Disease Control and Prevention. Differences by sex in tobacco use and awareness of tobacco marketing - Bangladesh, Thailand, and Uruguay, 2009. MMWR Morb Mortal Wkly Rep 2010; 59:613-8.

37. Malta DC, Moura EC, Silva SA, Oliveira PP, Silva VL. Prevalência do tabagismo em adultos residentes nas capitais dos estados e no Distrito Federal, Brasil, 2008. J Bras Pneumol 2010; 36:75-83.
38. Azevedo e Silva G, Valente JG, Malta DC. Tendências do tabagismo na população adulta das capitais brasileiras: uma análise dos dados de inquéritos telefônicos de 2006 a 2009. Rev Bras Epidemiol 2011; 14 Suppl 1:103-14.

39. Costa e Silva VL, Koifman S. Smoking in Latin America: a major public health problem. Cad Saúde Pública 1998; 14:99-108.

40. Mercado Común del Sur. Primer reporte de vigilancia de enfermedades no transmisibles (ENT): situación epidemiologica de las ENT y lesiones en Argentina, Brasil, Chile, Paraguay y Uruguay. Buenos Aires: Mercado Común del Sur; 2011.

41. Vargas MA, Campos RR. Crop substitution and diversification strategies: empirical evidence from selected Brazilian municipalities. Washington DC: The International Bank for Reconstruction Development, The World Bank; 2005.

42. Boseley S. Passive smoking kills 600,000 a year, in cluding 165,000 children, says WHO. The Guardian, 2010. http://www.guardian.co.uk/society/2010/ nov/26/passive-smoking-deaths-who-report (acessado em 16/Nov/2012).

43. Lilienfeld AM, Lilienthal DE. Foundations of epidemiology. New York: Oxford University Press; 1980.

44. Malta DC, Moura L, Souza MF, Curado MP, Alencar AP, Alencar GP. Tendência de mortalidade do câncer de pulmão, traquéia e brônquios no Brasil, 1980-2003. J Bras Pneumol 2007; 33:536-43.

45. Wilson LM, Tang EA, Chander G, Hutton HE, Odelola OA, Elf JL, et al. Impact of tobacco control interventions on smoking initiation, cessation, and prevalence: a systematic review. J Environ Public Health 2012; 2012:961724.

Recebido em 12/Ago/2012

Versão final reapresentada em 21/Nov/2012 Aprovado em 03/Dez/2012 